\title{
Epidemic Characteristics and Prevention and Control Implications of COVID-19 in China
}

\author{
Li Ming Quan \\ Nanchong City Committee Office of the Chinese People's Political Consultative Conference, Nanchong, China \\ Email address: \\ 617738477@qq.com \\ To cite this article: \\ Li Ming Quan. Epidemic Characteristics and Prevention and Control Implications of COVID-19 in China. European Journal of Preventive \\ Medicine. Vol. 9, No. 4, 2021, pp. 101-106. doi: 10.11648/j.ejpm.20210904.11
}

Received: June 18, 2021; Accepted: June 25, 2021; Published: July 8, 2021

\begin{abstract}
The COVID-19 epidemic is spreading around the world and has a serious impact on the economies of various countries. In order to promote the advanced experience of prevention and control of COVID-19 epidemic, the author wrote an article. Objective: To understand the occurrence and development of COVID-19 in China, and summarize the successful experience and inspiration of COVID-19 prevention and control in China. Methods: The epidemiological analysis of COVID-19 epidemic in China was carried out by using actual cases and data. Conclusion and discussion: The main characteristics and laws of the COVID-19 epidemic in China were revealed. The prevention and control of COVID-19 epidemic can effectively contain the rapid spread of the epidemic as long as the methods and measures of early detection and prevention are adopted. The transmission speed of COVID-19 virus is related to the number of infected people and the distance and population density of the introduced area. The farther away you are, the later you have the first case, the more people you introduce, the more people you get infected. The COVID - 19 virus susceptible object while regardless of age, gender, race, and occupation, but through the analysis found, 30-69 - year - old ages and male crowd COVID - 19 is higher than other age groups and the number of female population, which may be associated with them to participate in social activities frequently, the article summarizes the experience of prevention and control work, suggestions on prevention and control of major infectious diseases in the future were put forward.
\end{abstract}

Keywords: COVID-19, Epidemiological, Characteristics, Prevention and Control Enlightenment

\section{Introduction}

A large COVID-19 outbreak occurred in China from December 2019 until now. In late December 2019, an unexplained pneumonia occurred in Wuhan, China, which was later diagnosed as COVID-19 (abbreviated as COVID-19 in China, and the WHO named the novel coronavirus that caused pneumonia as COVID-19). The National Health Commission immediately disclosed the situation of the epidemic, reported the relevant data of the epidemic nationwide on a daily basis, actively took various measures to prevent the spread of the epidemic, and cooperated with relevant international organizations to carry out etiological and epidemiological investigations. The outbreak spread fast, spread wide, on January 23, 0 to 2020 in wuhan city, hubei province issued closure (wu) han channels marked, the national implementation of the response to major public health emergencies not I level emergency response, mobilize various forces play outbreak of total war, war, all over the country quickly if necessary in wuhan in hubei province, This is a major public health emergency that China has experienced since the founding of the People's Republic of China, with the fastest speed of transmission, the widest scope of infection and the greatest difficulty in preventing and controlling it. It is also the largest epidemic prevention and control campaign ever. Through painstaking efforts, the epidemic was soon put to rest. At present, the COVID-19 epidemic in China has been well controlled domestically, and the production and living order in various regions has been restored to normal, and economic activities have recovered to the pre-epidemic level. At present, all parts of China are actively doing a good job in the epidemic prevention and control work of "import from external prevention and rebound from internal prevention". In order to accumulate experience and draw lessons from the prevention and control of infectious diseases, the author studied and analyzed a large number of data to summarize and analyze the characteristics of the COVID-19 epidemic in China and its implications in the prevention and 
control of the epidemic. The report is as follows:

\section{Material Source and Finishing Method}

\subsection{Data Sources}

(1) Typical cases in the paper are extracted from open Internet media and public reports and disclosures of health commissions at all levels.

(2) All data sources that are not indicated are from the official website of the Health Commission, PRC.

\subsection{Data Analysis Method}

(1) Collected data shall be sorted out by biometric method and made into tables for data statistical analysis [1]

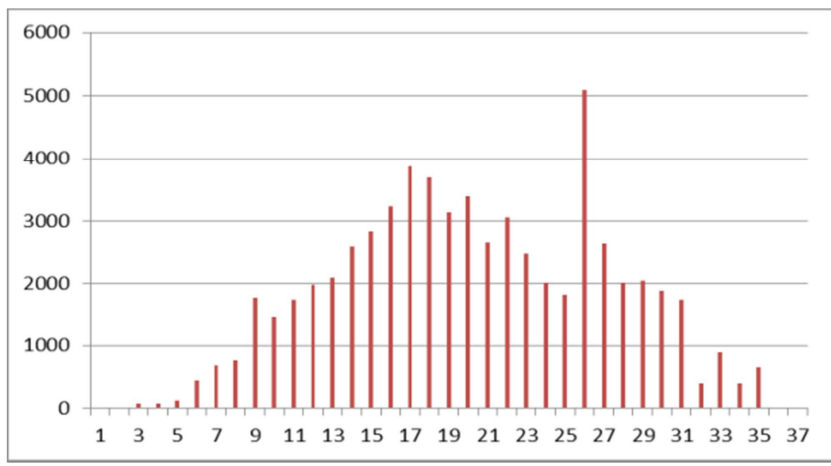

Figure 1. National chart of confirmed COVID-19 cases as of February 23, 2020.

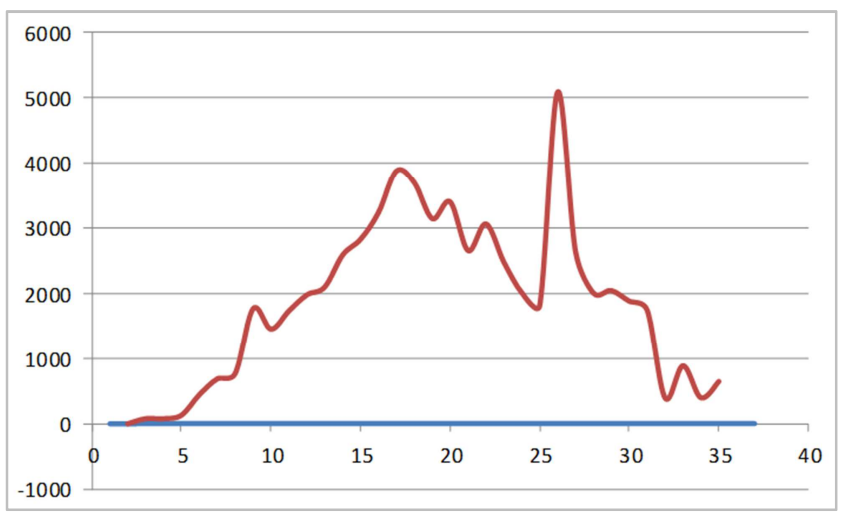

Figure 2. National chart of confirmed cases as of February 2, 2020.

(2) Difference analysis was conducted on the cases of this COVID-19 epidemic.

The correlation between the number of new cases in each province on the same day and the distance between Hubei province and the population of each province was analyzed to find out its rule. A large number of biometric data are calculated with the help of Excel data analysis function.

(3) Corresponding hypothesis test for the calculated parameters was adopted to determine the reliability of the parameters.

(4) The number is in chronological order in the situation analysis of the occurrence of the epidemic.

The time is the abscisate, and the newly confirmed cases are the ordinate. The histogram and change curve formed only represent the period of data collection.

Table 1. Statistical table of daily confirmed cases of COVID-19 from January 22, 2020 to February 24, 2020 Unit: case [2].

\begin{tabular}{|c|c|c|c|c|c|c|c|c|c|c|c|c|c|c|c|c|c|}
\hline Order no. & 1 & 2 & 3 & 4 & 5 & 6 & 7 & 8 & 9 & 19 & 11 & 12 & 13 & 14 & 15 & 16 & 17 \\
\hline New cases & 77 & 131 & 444 & 688 & 769 & 1771 & 1549 & 1737 & 1982 & 2102 & 2590 & 2829 & 3235 & 3887 & 3694 & 3143 & 3399 \\
\hline
\end{tabular}

Table 1. Continued

\begin{tabular}{llllllllllllllllll}
\hline Order no. & $\mathbf{1 8}$ & $\mathbf{1 9}$ & $\mathbf{2 0}$ & $\mathbf{2 1}$ & $\mathbf{2 2}$ & $\mathbf{2 3}$ & $\mathbf{2 4}$ & $\mathbf{2 5}$ & $\mathbf{2 6}$ & $\mathbf{2 7}$ & $\mathbf{2 8}$ & $\mathbf{2 9}$ & $\mathbf{3 0}$ & $\mathbf{3 1}$ & $\mathbf{3 2}$ & $\mathbf{3 3}$ & $\mathbf{3 4}$ \\
\hline New cases & 2656 & 3062 & 2478 & 2015 & 1820 & 5090 & 2641 & 2009 & 2048 & 1886 & 1749 & 394 & 889 & 397 & 648 & 409 & 508 \\
\hline
\end{tabular}

\section{Characteristics and Analysis}

\subsection{Basic Trend of COVID-19 Outbreak in China}

The COVID-19 outbreak occurred in Wuhan, Hubei Province in late December 2019, with pneumonia of unknown cause and no human-to-human transmission determined. Later, due to the spread of the epidemic, cases occurred in many places in Hubei Province, and a high-level expert group was sent to Wuhan for investigation, which confirmed COVID-19 and confirmed the case of human-to-human transmission. On January 20, 2020, Hubei Province announced the situation of the epidemic for the first time [2], saying that it had accumulated as of 24:00 on January 20, 2020 in Hubei Province Table 1 [2], Statistical table of daily confirmed cases of COVID-19 from January 22, 2019 to February 24, 2020

A total of 270 cases of pneumonia infected by novel coronavirus were reported, among which one doctor and two nurses in Huanggang City were infected [3]. 72 new cases were reported on the same day, 25 cases were cured and discharged from hospital, and 6 cases died. Announced by Wuhan Health Commission on January 19, 2020, as of, On January 17, 2020, 62 cases of novel coronavirus pneumonia have been reported in Wuhan, of which 19 cases have been cured and discharged, 8 cases are under severe treatment, and 2 cases have died [4]. In the face of the severe situation of the epidemic, the Wuhan People's Government has made a decision to close all the passages leaving Wuhan (Wuhan) and Han from 0:00 on January 23, 2020, to stop the outward flow of people in Wuhan and prevent the outward spread of the epidemic. At the same time, a number of provinces, autonomous regions and municipalities in China launched a level I emergency response to a major public health emergency, and the battle against COVID-19 was officially launched nationwide. According to the analysis of the outbreak, the first case in Hubei was reported in late December 2019. The first cases in other provinces were from 
early January 2020 to early February 2020, and the specific time range was January 4, 2020 to February 2, 2020. Statistics of the first case in 30 provinces (urban areas) were carried out from January 1 to January 9, 2020. The number of the first case was $16.67 \%$ in 5 provinces and urban areas. The first case occurred in 16 provinces, accounting for $53.33 \%$, from 10 th to 19th, and 9 provinces, accounting for $30 \%$, where the first case occurred from 20th January to 2nd February. The first case of COVID-19 was first reported in Zhejiang Province on January 4, 2020. The patient was a 46-year-old male who had lived in Wuhan for a long time. He drove from Wuhan to Wenzhou on January 3 and went to hospital on January 4 when he developed fever symptoms. Beijing is the last city in China to have the first case of COVID-19. The time is February 2, 2020. The patient, Ms. Chen, is from Xiaogan, Hubei Province, who works in Zhejiang Province and her husband works in Beijing. After returning to her hometown Xiaogan to stay with her children for a few days before the Spring Festival, Chen took a train to Beijing on December 29 to visit her lover. However, a few days after her reunion with her lover, Ms. Chen suddenly began to develop cold symptoms such as runny nose and headache on February 2. Then she developed a fever, with the highest temperature reaching $38.4^{\circ} \mathrm{C}$, and went to a doctor in Beijing [6]. It took 30 days from the first COVID-19 case in a few provinces to the first COVID-19 case in the whole country. For each province, the first cases in 30 provinces were imported from Wuhan or other cities in Hubei. The first case in Shandong province was a 37-year-old male from Wuhan who worked in the city of Rizhao, although he had no direct contact with people in Wuhan. She went to see a doctor in Rizhao City on January 17 due to fever and other symptoms, and went to see a doctor in Qingdao City on her own that night. Through pre-examination and triage, it was learned that she had lived in Wuhan within two weeks before the onset of illness [7].

From the first Figure 1: Curve of confirmed cases of COVID-19 in China as of February 23, 2020 Figure 2: Curve of confirmed cases in China as of February 2, 2020. All cases had contact history with Hubei Province, especially Wuhan population. As of February 23, 2020, the number of new COVID-19 diagnoses nationwide on A daily basis is summarized in Table 1, and the trend charts are plotted in Figure 1 and Figure 2. As can be seen from the above chart, the number of confirmed cases of the epidemic has been increasing day by day since January 23, 2020, when the national public health emergency level I response was implemented, indicating that the epidemic is spreading continuously and the infection degree is deepening. The epidemic reached its peak on February 4 and 5, 2020, with 3,877 and 3,694 confirmed cases, respectively. Since then, the confirmed cases showed a fluctuating trend of decline, indicating that the emergency response measures were effective. At the same time, the statistical caliber of Hubei Province was changed on February 12 and 13, and clinically confirmed cases were suddenly increased. The number of confirmed cases on February 12 reached 15,152. Later, the data were revised according to the relevant technical requirements, and 13, 332 clinically confirmed cases were subtracted from the number of confirmed cases on February 12. The number of confirmed cases on 12 and 13 were 1820 and 5090, respectively, so the graph shows a clear peak image (the number in the statistical table corresponds to serial number 23, and the time is February 13, 2020). By February 25,2020 , the number of newly confirmed cases in China had dropped to 508, and 530 suspected cases had been added on that day, with Hubei accounting for $70-80 \%$. The momentum of the epidemic had been effectively contained. From February 22 to February 26, the number of newly confirmed cases except Hubei Province had been less than 10. Many provinces, autonomous regions and municipalities have generally downgraded their emergency response levels from Level I to II or lower.

\subsection{Vulnerable Populations}

World Health Organization experts sequenced the whole genome of 104 novel coronavirus strains isolated from different sites, and confirmed that the homology reached $99.9 \%$, suggesting that the virus has not yet undergone significant mutation. Bats may be the host of novel coronavirus, and pangolins may be one of the intermediate hosts of virus. According to the analysis of reported cases and epidemic situation across the country, the average age of patients is 51 years old, and nearly $78 \%$ of the cases are from Hubei Province [8]. The novel coronavirus infection is mainly human, so far no other animal large-scale infection of the novel coronavirus has been reported. COVID-19 is transmitted mainly through droplets and contact. According to the age analysis of the patients, there is no specific age segment of the infected patients, and some of the patients are older than 90 years old, and some of the infants are less than 1 year old. [9] Liu Y, Wang Y, Wang Y, et al. Novel coronavirus infection in a novel coronavirus pneumonia case [J]. J Clin Endocrinol, 2014, 25 (1): 87-92. However, of the 34,755 confirmed cases reported nationwide, $77.8 \%$ are in the age group of 30-69. The 30-69 age group is the main labor force in both urban and rural areas, with frequent contact with the outside world and fast mobility. Therefore, the prevention, control and management of this group should be especially strengthened in prevention and control. From the perspective of gender analysis of patients, COVID-19 disease can be induced by novel coronavirus in both males and females regardless of gender. Gender analysis of confirmed cases reported in multiple provinces and regions showed no significant difference in $\mathrm{X}^{2}$ test. However, a total of 34,755 confirmed cases were reported nationwide, including 17,684 male cases, accounting for $51.4 \%$ of the total cases, and 16,891 female cases, accounting for $48.6 \%$ of the total cases. The biometric $\mathrm{X}^{2}$ analysis showed that $\mathrm{X}^{2}$ $=10.776>X^{2} \quad 0.01=6.63$. There was a significant difference $(\mathrm{P}<0.01$.). There is no clear occupational trend in terms of the occupation of the patients. According to the analysis of the first cases in all districts and cities across the country, the victims include workers, farmers, migrant workers, government staff, students, teachers, travelers to the foci, 
staff at conferences, and doctors and nurses.

\subsection{Correlation Analysis Between COVID-19 Cases and the Population Size of the Province and the Distance Between the Province and Hubei Province}

In order to understand the transmission and pathogenic rules of COVID-19 infection, the relationship between COVID-19 transmission distance and the population of the imported province, region and city, What we have confirmed cases (in February 2020, 10 provinces (city) health construction commission's website data as the analysis object) with the provinces area (city) and the distance of Hubei province (Hubei province government car distance between stations and other provincial government stations) and the correlation of disease occurs between provincial population is analyzed, after finishing the relevant data is shown in table 2 . After a biostatistical analysis.

Table 2. Confirmed cases in provinces (autonomous regions and municipalities) and customers in the importing place. Population statistics unit: example, 10,000 people, $\mathrm{km}$.

\begin{tabular}{|c|c|c|c|c|c|c|c|c|c|c|c|c|c|c|c|}
\hline serial number & 1 & 2 & 3 & 4 & 5 & 6 & 7 & 8 & 9 & 10 & 11 & 12 & 13 & 14 & 15 \\
\hline cases & 1092 & 95 & 295 & 486 & 108 & 81 & 378 & 213 & 83 & 18 & 1 & 55 & 49 & 215 & 60 \\
\hline population & 2171 & 1557 & 2418 & 3048 & 4369 & 2717 & 3789 & 3835 & 2626 & 598 & 337 & 2445 & 682 & 4885 & 2529 \\
\hline distance & 1189 & 1150 & 814 & 938 & 1826 & 2112 & 2376 & 821 & 1570 & 1626 & 3116 & 3323 & 1502 & 1240 & 1566 \\
\hline
\end{tabular}

Table 2. Continued.

\begin{tabular}{|c|c|c|c|c|c|c|c|c|c|c|c|c|c|c|c|}
\hline serial number & 16 & 17 & 18 & 19 & 20 & 21 & 22 & 23 & 24 & 25 & 26 & 27 & 28 & 29 & 30 \\
\hline cases & 912 & 844 & 1105 & 239 & 860 & 149 & 118 & 417 & 1092 & 492 & 1159 & 267 & 157 & 38 & 122 \\
\hline population & 6820 & 4622 & 9559 & 7519 & 6255 & 4801 & 3580 & 8302 & 5657 & 8029 & 11169 & 3911 & 926 & 10006 & 3702 \\
\hline distance & 390 & 370 & 560 & 972 & 434 & 1604 & 1046 & 1156 & 748 & 560 & 1015 & 909 & 1557 & 866 & 1022 \\
\hline
\end{tabular}

There was a positive correlation between the population and the number of COVID-19 cases in each province $\left(\mathrm{r}_{12}=0.632\right.$, $\mathrm{P}<0.05$ ), and a negative correlation between the number of COVID-19 cases in each province $r_{13}=-0.571$ ) and the distance between the people's government residences and Hubei province $\left(\mathrm{r}_{13}=-0.571\right)$, indicating a significant correlation. That is to say, the farther away from the foci, the smaller the population and the fewer cases of COVID-19. The closer to the foci, the larger the population, and the more COVID-19 cases occurred. Relevant parameters of statistical analysis are shown in Table 3.

Table 3. Correlation analysis results of the confirmed cases with the distance to the importing place and the population of the affected province.

\begin{tabular}{|c|c|c|c|c|}
\hline Confirmed cases and factors $r_{1}$ & The correlationcoefficientr ${ }_{1, n}$ & Inspectiont value & The value of $t_{0,05}$ & Significance decision \\
\hline population of affected provinces and municipalities $r_{2}$ & 0.632 & 4.242 & 2.92 & Significant differences \\
\hline $\begin{array}{l}\text { The distance between the provinces and Hubei } \\
\text { Provincer }\end{array}$ & -0.571 & 3.609 & 2.92 & Significant differences \\
\hline
\end{tabular}

\section{Results and discussion}

\subsection{Since the End of 2019, the COVID-19 Pneumonia Epidemic Has Been Widespread in CHINA}

There have been confirmed cases in 31 provinces (autonomous regions and municipalities), and 1,396 counties (districts and municipalities) have confirmed cases, accounting for $46 \%$ of the total counties (districts and municipalities). In China, 1,639 counties, districts and municipalities had no confirmed cases, accounting for 54 percent of the total. It took 30 days from the first confirmed case in the first province to the first confirmed case in all provinces. According to the statistics of the time of the first case in 30 provinces and municipalities, 16 provinces and municipalities had the first case on January 10 to 19,2020 , accounting for $53.33 \%$. Therefore, the implementation of emergency response to public health emergencies before January 10 and taking measures will have better effect. Based on current epidemiology investigation, its incubation period is 3--7 days commonly, the longest is 24 days, incubation period has infectivity. The daily confirmed cases of the epidemic reached the peak of 3,877 and 3,694 cases on February 4 and 5 , 2020, respectively. Since February 5, the daily confirmed cases have fluctuated and decreased, and the statistical data fluctuated on February 12 due to the change of statistical coverage of the epidemic in Hubei Province. The National Health Commission quickly standardized the statistical caliber. By February 27, 2020, the number of newly confirmed cases in China had dropped to 327 , and the number of newly confirmed cases in China had been less than 10 for several consecutive days, with the exception of Hubei Province. The total number of discharged cases in China was 36,117 , and the number of discharged patients reached 3622 on the same day. The epidemic situation across the country began to stabilize and improve after recovery.

\subsection{We Analyzed the Related Factors Affecting the Occurrence of Novel Coronavirus Cases}

Since the new coronavirus pneumonia outbreak in China was first reported in Wuhan, Hubei Province, the confirmed cases in other provinces and municipalities were either infected with COVID-19 after returning from a business trip to Wuhan or transmitted back to others, or the first cases were 
caused by people in Wuhan directly carrying the virus. Poisoned for to take the same means of transport, the flow speed and the distance to its destination, the risk of infection to others how many associated with defined its population, and to analyze them with the provinces (area city) the relevance of the confirmed cases occurred, we to the provinces (area city) the confirmed cases and the distance from Hubei province, provincial population were analyzed related disease occur. Through analysis, it can be seen that the number of confirmed cases in provinces (autonomous regions and municipalities) is positively correlated with the total population of the province where the confirmed cases are located, and the correlation coefficient $\mathrm{R} 12=0.632$. The number of confirmed cases in provinces (autonomous regions and municipalities) is negatively correlated with the distance of Hubei Province, and the correlation coefficient R13=-0-571. The degree of correlation between them all reached a significant level $(\mathrm{P}<$ $0.05)$, that is, the number of confirmed cases in each province (autonomous region or municipality) was negatively correlated with the distance from Wuhan, Hubei Province, and the farther the distance was, the less the number of cases was. The number of confirmed cases in each province is positively correlated with the population of the province, and the larger the population, the greater the number of confirmed cases.

\subsection{The COVID-19 Epidemic in China Is Fierce, Fast in Transmission and Has a Wide Range of Effects}

However, thanks to the great attention paid by the Chinese governments at all levels and the correct prevention and control measures taken, the epidemic was quickly brought under control. According to the official website of the National Health Commission, from January 22, 2020, when the epidemic statistics were released, to March 2, 2020, there were 41 days, and the confirmed cases fluctuated from January 22, BBB 0, to February 4, a total of 14 days, indicating that the epidemic was spreading. Since Feb. 5, on the day of the new confirmed cases falling volatility and take various measures to strong and effective, the speed of the spread of the virus has been curbed, the country will be coronavirus disease control effect appeared gradually, to the National Day on March 3, 2020 cases of the new fell 38 days, the National Day on March 3, the new COVID - 19 confirmed disease in 125 cases, 31 cases deaths, Except for the hardest-hit Hubei province, the death rate dropped to zero on that day, and the recovery has been positive, and the number of people cured and discharged from hospital is in the thousands every day. The epidemic has been brought under effective control, and the economy and society have gradually returned to normal order. This is one of the major infectious diseases prevention and control of wonders, also gave us a lot of inspiration: one is the Chinese system is superior, the efficient governance mechanism, in a very short time launched a nationwide public health emergencies emergency response grade $\mathrm{I}$, in a short period of time established a longitudinal, transverse to the edge of the epidemic prevention and control network, kicked off the national scope, the outbreak unfolds. Second, we put the people first and life first.
We did everything for the health and safety of the people. We drew on the enthusiasm of the general public for fighting the epidemic, and soon gathered a mighty force to fight the epidemic. Third, we need to concentrate our efforts to accomplish major tasks. After the outbreak of the epidemic, under the unified coordination of Party committees and governments, we effectively ensured the supply of prevention and control materials, especially in the worst-hit areas, laying a foundation for winning the fight against the epidemic. Fourth, we will coordinate emergency response efforts of technical forces. While preventing the spread, we will screen effective drugs for symptomatic treatment, improve prevention and control measures as well as the treatment and treatment of patients, share best experience in prevention, control and treatment of the epidemic across the country, and do our best to cure patients and save lives. Fifth, the spirit of the nation and the confidence of the whole people have laid a strong ideological foundation for the fight against the epidemic.

\subsection{COVID-19 Is Transmitted in Humans and Causes Pneumonia}

All humans are susceptible regardless of region and ethnic group, age, gender and occupation. However, the number of cases in a given age varies, and the number of confirmed cases varies between men and women. In terms of age of onset, $77.8 \%$ of the 34,775 confirmed cases in China were aged between 30 and 69 years old.

\section{Conclusion}

Based on a large number of data analysis, this paper demonstrates that the prevention and control of COVID-19 epidemic can effectively contain the rapid spread of the epidemic as long as the methods and measures of early detection and prevention are adopted [10]. The transmission speed of COVID-19 virus is related to the number of infected people and the distance and population density of the introduced area. The farther away you are, the later you have the first case, the more people you introduce, the more people you get infected. Although the susceptible subjects of COVID-19 virus were not divided into age, gender, race and occupation, the analysis showed that the number of people aged 30-69 and men with COVID-19 was higher than other age groups and women, which may be related to their frequent participation in social activities. At present, the COVID-19 epidemic in China has been completely controlled, and people's production and life have already returned to normal. Economic activity has returned to pre-outbreak levels. Countries with high levels of economic development and other severe epidemics. China's successful experience in COVID-19 prevention and control is as follows; (i) China has a superior social system and efficient governance mechanism [11]; (ii) Adhere to the concept of people first and life first for prevention and control [12]; (iii) Focusing on major issues has laid a strong material foundation for the prevention and control of the epidemic 
[13]. (iv) To tackle key problems in emergency response and solve new difficulties and problems in the prevention and control of the epidemic in a timely manner [14]; (v) The traditional culture of "when one side is in trouble, there will be support from all sides" has inspired a mighty force in the fight against the epidemic. [15]

\section{References}

[1] Ming Daoxu, Field Experiment and Statistical Analysis, 2nd edition M Science Press.

[2] Notify the official website of the Health Commission, PRC of the epidemic situation.

[3] Inform the official website of Hubei Provincial Health Commission of the epidemic situation.

[4] The official website of Wuhan Health Commission was notified of the epidemic situation.

[5] The epidemic was notified to Wenzhou Center for Disease Control and Prevention.

[6] Beijing Daily Client February 15, 2020.

[7] Shandong Education News Network February 17, 2020.
[8] World Health Organization expert investigation conclusion CCTV network (news broadcast) February 24, 2020.

[9] The epidemic was notified to the Health Commission of Jiangsu Province on February 5, 2020.

[10] White Paper "China's Action against COVID-19" (1) Xinhua official account 2020.4.8.

[11] Dong Xinhao, Hu Hao, Jiang Yile, Li Feng, et al. The New Pneumonia Epidemic and the World Economy www.china.cer.com.cn 2020.4.8.

[12] Xi Jinping, Speech at the Commendation Conference on the National Fight against COVID-19 Epidemic, Qiushi, 2020.10.15.

[13] Zhou Jin, "The Experience and Enlightenment of China's Fight against COVID-19 Epidemiology under the Global Perspective", Journal of World Socialism, 2020.5.01, P41-50+93-94.

[14] China's experience in combating the COVID-19 epidemic on CNR.com provides a useful reference for the world to fight the epidemic.

[15] Xu Da-wei, The institutional advantages and practical experience of epidemic prevention in China, Theoretical construction, online release. 